\title{
McDonald Generalized Linear Failure Rate Distribution
}

\author{
Ibrahim Elbatal \\ Institute of Statistical Studies and Research \\ Department of Mathematical Statistics \\ Cairo University, Egypt \\ i_elbatal@staff.cu.edu.eg, i_elbatal@yahoo.com \\ Faton Merovci \\ Department of Mathematics \\ University of Prishtina "Hasan Prishtina" \\ Republic of Kosovo, Albania \\ fmerovci@yahoo.com \\ W. Marzouk \\ Institute of Statistical Studies and Research \\ Department of Mathematical Statistics \\ Cairo University, Egypt \\ wgm-74@hotmail.com
}

\begin{abstract}
We introduce in this paper a new six-parameters generalized version of the generalized linear failure rate (GLFR) distribution which is called McDonald Generalized Linear failure rate (McGLFR) distribution. The new distribution is quite flexible and can be used effectively in modeling survival data and reliability problems. It can have a constant, decreasing, increasing, and upside down bathtub-and bathtub shaped failure rate function depending on its parameters. It includes some well-known lifetime distributions as special sub-models. Some structural properties of the new distribution are studied. Expressions for the density, moment generating function, conditional moments, mean deviation, Bonferroni and Lorentz curves also are obtained. Moreover we discuss maximum likelihood estimation of the unknown parameters of the new model.
\end{abstract}

Keywords: Generalized Linear Failure Rate, Moment generating function, Moments, Maximum likelihood estimation.

\section{Introduction}

In analyzing lifetime data one often uses the exponential, Rayleigh, linear failure rate or generalized exponential distributions. It is well known that exponential can have only constant hazard function whereas Rayleigh, linear failure rate and generalized exponential distribution can have only monotone (increasing in case of Rayleigh or linear failure rate and increasing decreasing in case of generalized exponential distribution) hazard functions. Unfortunately, in practice often one needs to consider non-monotonic function such as bathtub shaped hazard function also, see, for example, Lai et al. (2001). The Generalized linear failure rate distribution generalizes both these distributions which may have non-increasing hazard function also. Also, the Weibull distribution, having the exponential, Rayleigh as special cases,is very popular distribution for modeling lifetime data and for modeling phenomenon with monotone failure rates, when modeling monotone hazard rates, the Weibull distribution may be an initial choice because of its negatively and positively skewed density shapes. However, the Weibull distribution does 
not provide a reasonable parametric fit for modeling phenomenon with non-monotone failure rates such as the bathtub-shaped and the unimodal failure rates which are common in reliability and biological studies. The models that present bathtub-shaped failure rate are very useful in survival analysis. But, according to Nelson (1982), the distributions presented in lifetime literature with this type of data, as the distributions proposed by (1980), are sufficiently complex and, therefore, difficult to be modeled. Later, other works had introduced new distributions for modeling bathtub-shaped failure rate. For example, Rajarshi, S., Rajarshi, M (1988) presented a review of these distributions and Haupt and Schabe (1992) considered a lifetime model with bathtub failure rates.

Sarhan and Kundu (2009) introduced generalized linear failure rate , the new distribution due to its flexibility in accommodating all the forms of the hazard rate function can be used in a variety of problems for modeling lifetime data. Another important characteristic of the distribution is that it contains, as special sub-models, the generalized exponential distribution, generalized Rayleigh, Linear failure rate, exponential, and Rayleigh distributions.

A random variable $X$ is said to have the generalized linear failure rate distribution $(G L F R)$ with three parameters $(\alpha, \beta, \theta)$ if its probability density function is given by

$$
g(x)=\theta(\alpha+\beta x) e^{-\left(\alpha x+\frac{\beta}{2} x^{2}\right)}\left[1-e^{-\left(\alpha x+\frac{\beta}{2} x^{2}\right)}\right]^{\theta-1}, \alpha, \beta, \theta>0,
$$

while the cumulative distribution function is given by

$$
G(x, \alpha, \beta, \theta)=\left[1-e^{-\left(\alpha x+\frac{\beta}{2} x^{2}\right)}\right]^{\theta}, \alpha, \beta, \theta>0 .
$$

where $\alpha, \beta$ are scale parameters of the distribution whereas the parameter $\theta$ denotes the shape parameters.

The aim of this paper is extend the $(G L F R)$ distribution by introducing three extra shape parameters to define a new distribution refereed to as the McDonald generalized linear failure rate $(M c G L F R)$ distribution. The role of the three additional parameters is to introduce skewness and to vary tail weights and provide greater flexibility in the shape of the generalized distribution and consequently in modeling observed data. It may be mentioned that although several skewed distribution functions exist on the positive real axis, not many skewed distributions are available on the whole real line, which are easy to use for data analysis purpose. The main idea is to introduce three shape parameters, so that the (McGLFR) distribution can be used to model skewed data, a feature which is very common in practice.

\subsection{Mc-Donald Generalized Distribution}

For an arbitrary parent cdf $G(x)$. The probability density function (pdf) $f(x)$ of the new class of distributions called the Mc-Donald generalized distributions (denoted with the prefix " Mc" for short) is defined by

$$
f(x, a, b, c)=\frac{c}{B(a, b)} g(x)[G(x)]^{a c-1}\left[1-(G(x))^{c}\right]^{b-1},
$$


where $a>0, b>0$ and $c>0$ are additional shape parameters . ( See Corderio et al. (2012) for additional details). Note that $g(x)$ is the pdf of parent distribution, $g(x)=\frac{d G(x)}{d x}$. The class of distributions (3) includes as special sub-models the beta generalized (Eugene et al. (2002)) for $c=1$ and Kumaraswamy (Kw) generalized distributions (Cordeiro \& Castro, (2011)) for $a=1$. For random variable $X$ with density function (3), we write $X \sim M c-G(a, b, c)$. The probability density function (3) will be most tractable when $G(x)$ and $g(x)$ have simple analytic expressions. The corresponding cumulative function for this generalization is given by

$$
F(x, a, b, c)=I_{G^{c}(x)}(a, b)=\frac{1}{B(a, b)} \int_{0}^{G(x)^{c}} w^{(a-1)}(1-w)^{b-1} d w
$$

where $I_{y}(a, b)=\frac{1}{B(a, b)} \int_{0}^{y} w^{(a-1)}(1-w)^{b-1} d w$ denotes the incomplete beta function ratio (Gradshteyn \& Ryzhik, (2000)). Equation (4) can also be rewritten as follows

$$
F(x, a, b, c)=\frac{G(x)^{a c}}{a B(a, b)_{2}} F_{1}\left(a, 1-b ; a+1 ; G(x)^{c}\right),
$$

where

$$
{ }_{2} F_{1}(a, b ; c ; x)=B(b, c-b)^{-1} \int_{0}^{1} \frac{t^{b-1}(1-t)^{c-b-1}}{(1-t x)^{a}} d t
$$

is the well-known hypergeometric functions which are well established in the literature (see, Gradshteyn and Ryzhik (2000)). Some mathematical properties of the cdf $F(x)$ for any Mc- G distribution defined from a parent $G(x)$ in equation (5), could, in principle, follow from the properties of the hypergeometric function, which are well established in the literature (Gradshteyn and Ryzhik, 2000, Sec. 9.1). One important benefit of this class is its ability to model skewed data that cannot properly be fitted by many other existing distributions. Mc- G family of densities allows for higher levels of flexibility of its tails and has a lot of applications in various fields including economics, finance, reliability, engineering, biology and medicine.

The hazard function (hf) and reverse hazard functions (rhf) of the Mc-G distribution are given by

$$
h(x)=\frac{f(x)}{1-F(x)}=\frac{c g(x) G^{a c-1}(x)\left[1-G^{c}(x)\right\}^{b-1}}{B(a, b)\left\{1-I_{G^{c}(x)}(a, b)\right\}},
$$

and

$$
\tau(x)=\frac{f(x)}{F(x)}=\frac{c g(x) G^{a c-1}(x)\left[1-G^{c}(x)\right]^{b-1}}{B(a, b)\left\{I_{G^{c}(x)}(a, b)\right\}},
$$

respectively. Recently Cordeiro et al. (2012) presented results on the McDonald normal distribution, Cordeiro et al. (2012) proposed McDonald Weibull distribution, and Francisco et al. (2012) obtained the statistical properties of the $M c-\Gamma$ and applied the model to reliability data. Oluyede and Rajasooriya (2013) introduced the Mc-Dagum distribution and its Statistical Properties with Applications. 
The rest of the article is organized as follows. In Section 2, we define the cumulative, density and hazard functions of the McGLFR distribution and some special cases. Section 3 includes the statistical properties such as $r_{t h}$ moment, moment generating function . The distribution of the order statistics are proposed in Section 4. Least squares and weighted least squares estimators introduced in Section 5. Finally, maximum likelihood estimation of the parameters is determined in Section 6.

\section{McDonald Generalized Linear Failure Rate Distribution}

In this section we studied the seven parameter McDonald generalized linear failure rate (McGLFR) distribution. Using $G(x)$ and $g(x)$ in (3) to be the cdf and pdf of (1) and (2). The pdf of the $M c G L F R$ distribution is given by

$$
f(x, \varphi)=\frac{c \theta(\alpha+\beta x)}{B(a, b)} e^{-\left(\alpha x+\frac{\beta}{2} x^{2}\right)}\left[1-e^{-\left(\alpha x+\frac{\beta}{2} x^{2}\right)}\right]^{\theta a c-1}\left\{1-\left[1-e^{-\left(\alpha x+\frac{\beta}{2} x^{2}\right)}\right]^{\theta c}\right\}^{b-1}
$$

where $\alpha, \beta$ are scale parameters the other positive parameters $\theta, a, b$ and $c$ are shape parameters, and $\varphi=(x, \alpha, \beta, \theta, a, b, c)$. The corresponding cdf of the McGLFR distribution is given by

$$
\begin{aligned}
F(x) & =I_{G^{c}(x)}(a, b)=\frac{1}{B(a, b)} \int_{0}^{G(x)^{c}} w^{(a-1)}(1-w)^{b-1} d w \\
& =\frac{1}{B(a, b)} \int_{0}^{\left.-\left(\alpha x+\frac{\beta}{2} x^{2}\right)\right)^{\theta c}} w^{(a-1)}(1-w)^{b-1} d w \\
& \left.=I_{(1-e}-\left(\alpha x+\frac{\beta}{2} x^{2}\right)\right)^{\theta c}(a, b),
\end{aligned}
$$

also, the cdf can be written as follows

$$
F(x)=\frac{\left(1-e^{-\left(\alpha x+\frac{\beta}{2} x^{2}\right)}\right)^{a \theta c}}{a B(a, b)} F_{1}\left(a, 1-b ; a+1 ;\left(1-e^{-\left(\alpha x+\frac{\beta}{2} x^{2}\right)}\right)^{\theta c}\right),
$$

where ${ }_{2} F_{1}(a, b ; c ; x)=B(b, c-b)^{-1} \int_{0}^{1} \frac{t^{b-1}(1-t)^{c-b-1}}{(1-t x)^{a}} d t$.

Figures 0 and 1 illustrates some of the possible shapes of the pdf and cdf of the McGLFR distribution for selected values of the parameters $a, b, c, \alpha, \beta$ and $\theta$, respectively. 


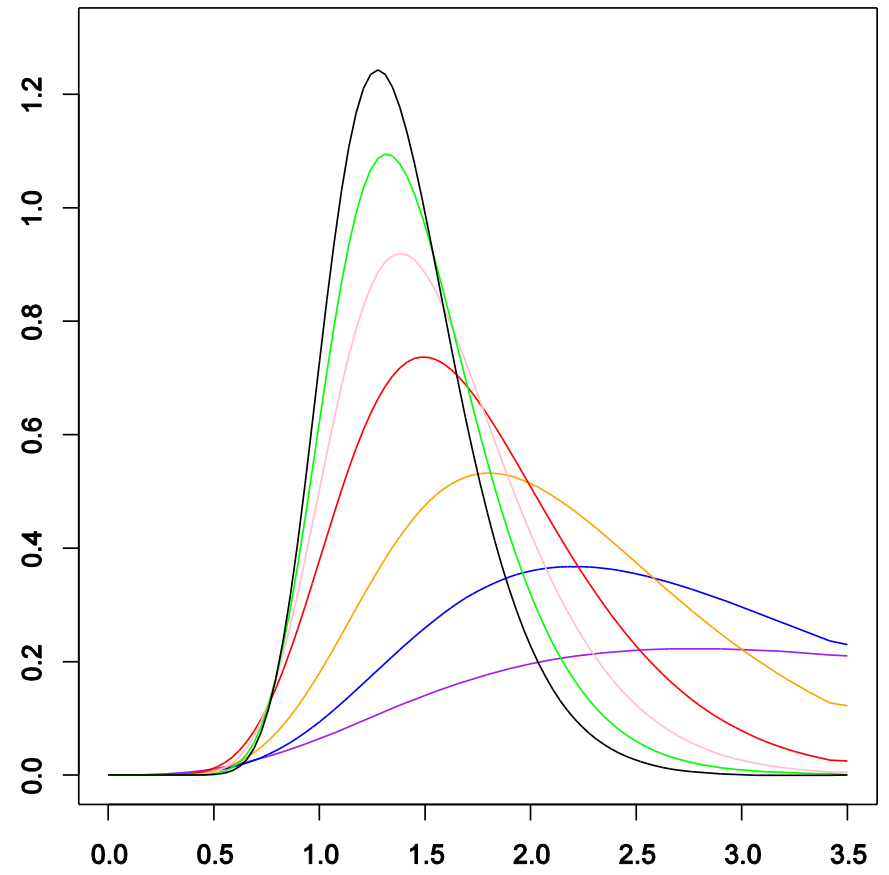

Figure 1: The pdf's of various McGLFR distributions for values of parameters:

$$
\begin{aligned}
& \alpha=0.5 ; 0.3 ; 0.4 ; 0.5 ; 0.7 ; 0.8 ; 0.9 ; \beta=1 ; 1.2 ; 1.5 ; 2 ; 2.2,2.4 ; 2.5, \theta=2.5, a=2.5, \\
& b=0.1 ; 0.2 ; 0.3 ; 0.4 ; 0.5 ; 0.6 ; 0.7 ; c=0.5 ; 0.6 ; 0.8 ; 1 ; 1.5 ; 2 ; 2.5
\end{aligned}
$$

with color shapes purple, blue, orange, red, pink, green and black, respectively.

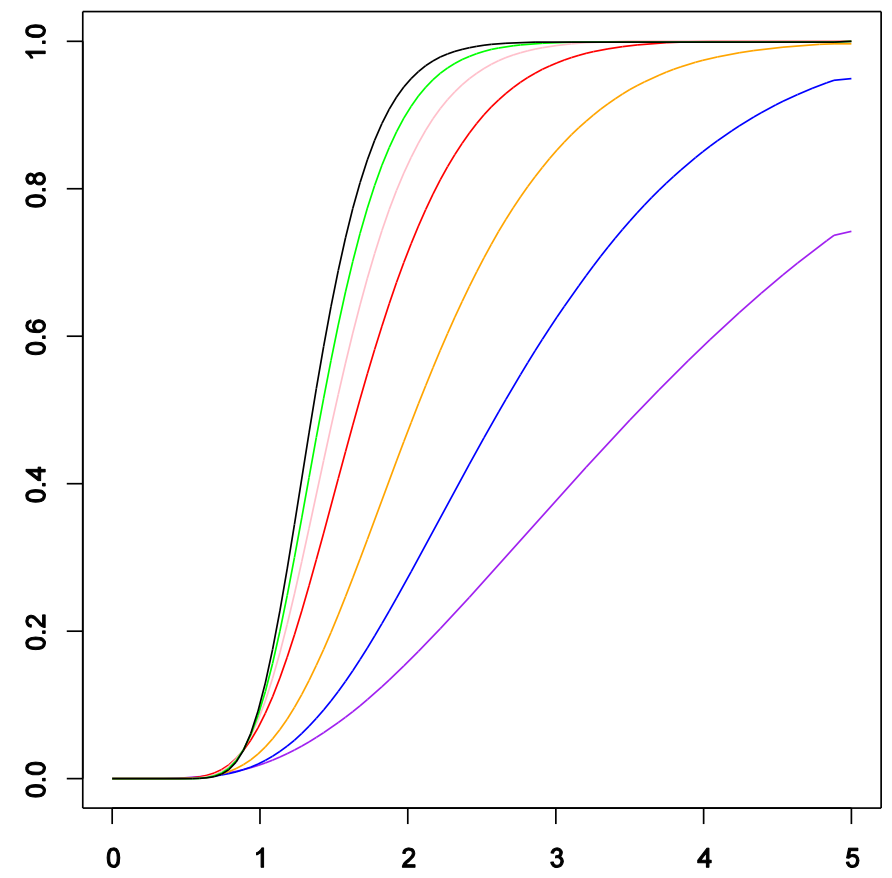

Figure 2: The pdf's of various McGLFR distributions for values of parameters:

$$
\begin{aligned}
& \alpha=0.5 ; 0.3 ; 0.4 ; 0.5 ; 0.7 ; 0.8 ; 0.9 ; \beta=1 ; 1.2 ; 1.5 ; 2 ; 2.2,2.4 ; 2.5, \theta=2.5, a=2.5, \\
& b=0.1 ; 0.2 ; 0.3 ; 0.4 ; 0.5 ; 0.6 ; 0.7 ; c=0.5 ; 0.6 ; 0.8 ; 1 ; 1.5 ; 2 ; 2.5
\end{aligned}
$$

with color shapes purple, blue, orange, red, pink, green and black, respectively. 
The hazard rate function and reversed hazard rate function of the new distribution are given by

$$
h(x)=\frac{f(x, \varphi)}{1-F(x, \varphi)}
$$

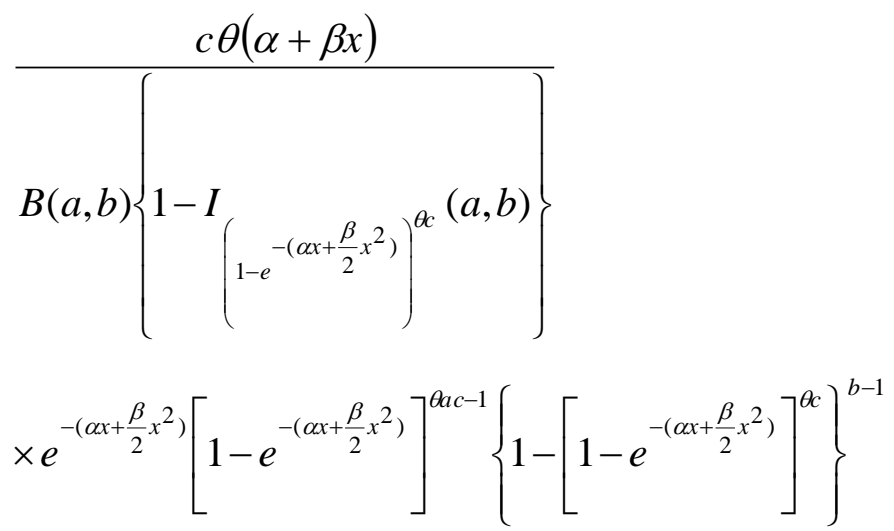

and

$$
\begin{aligned}
\tau(x) & =\frac{f(x, \varphi)}{F(x, \varphi)} \\
& =\frac{c \theta(\alpha+\beta x)}{B(a, b)\left\{I\left(1-e^{-\left(\alpha x+\frac{\beta}{2} x^{2}\right)}\right)^{\theta c}(a, b\}\right.} \\
& \times e^{-\left(\alpha x+\frac{\beta}{2} x^{2}\right)}\left[1-e^{-\left(\alpha x+\frac{\beta}{2} x^{2}\right)}\right]^{\theta a c-1}\left\{1-\left[1-e^{-\left(\alpha x+\frac{\beta}{2} x^{2}\right)}\right]^{\theta c}\right\}^{b-1},
\end{aligned}
$$

respectively.

Figures 3 and 4 illustrates some of the possible shapes of the survival funvction and hazard function of the McGLFR distribution for selected values of the parameters $a, b, c, \alpha, \beta$ and $\theta$, respectively. 


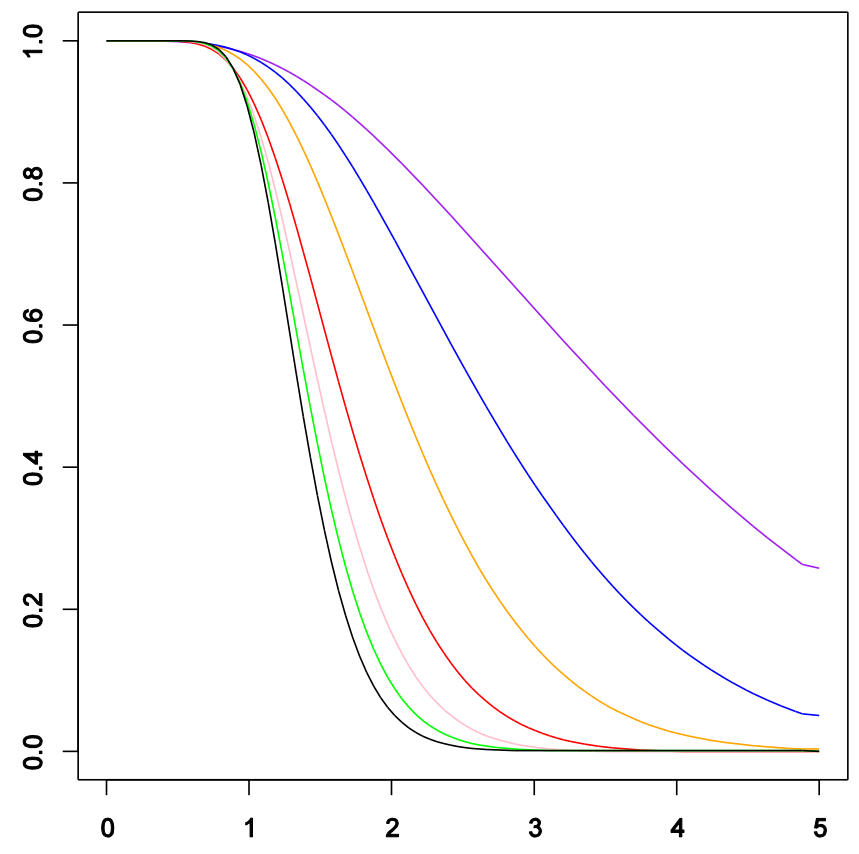

Figure 3: The survival function's of various McGLFR distributions for values of parameters:

$$
\begin{aligned}
& \alpha=0.5 ; 0.3 ; 0.4 ; 0.5 ; 0.7 ; 0.8 ; 0.9 ; \beta=1 ; 1.2 ; 1.5 ; 2 ; 2.2,2.4 ; 2.5, \theta=2.5, a=2.5, \\
& b=0.1 ; 0.2 ; 0.3 ; 0.4 ; 0.5 ; 0.6 ; 0.7 ; c=0.5 ; 0.6 ; 0.8 ; 1 ; 1.5 ; 2 ; 2.5
\end{aligned}
$$

with color shapes purple, blue, orange, red, pink, green and black, respectively.

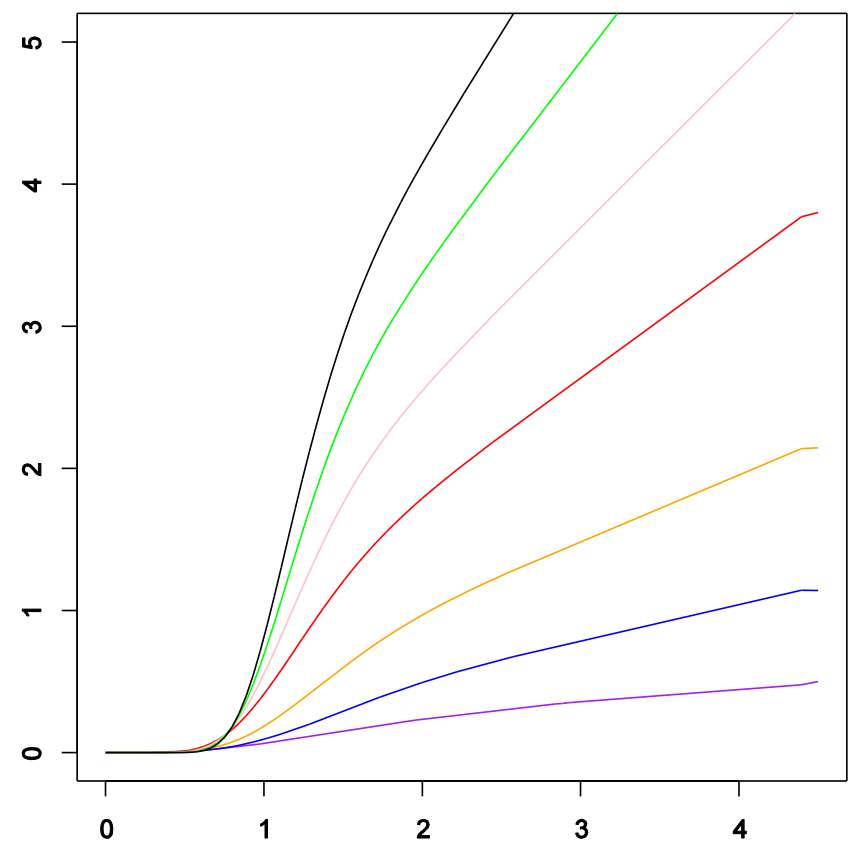

Figure 4: The hazard function's of various McGLFR distributions for values of parameters:

$$
\begin{aligned}
& \alpha=0.5 ; 0.3 ; 0.4 ; 0.5 ; 0.7 ; 0.8 ; 0.9 ; \beta=1 ; 1.2 ; 1.5 ; 2 ; 2.2,2.4 ; 2.5, \\
& \theta=2.5, a=2.5, b=0.1 ; 0.2 ; 0.3 ; 0.4 ; 0.5 ; 0.6 ; 0.7 ; c=0.5 ; 0.6 ; 0.8 ; 1 ; 1.5 ; 2 ; 2.5
\end{aligned}
$$

with color shapes purple, blue, orange, red, pink, green and black, respectively. 


\subsection{Submodels}

The McDonald generalized linear failure rate $(M c G L F R)$ distribution is very flexible model that approaches to different distributions when its parameters are changed. The McGLFR distribution contains as special- models the following well known distributions. If $X$ is a random variable with pdf (7), using the notation $X \sim M c-G((\alpha, \beta, \theta, a, b, c)$ then we have the following cases.

- Generalized Linear Failure Rate distribution: For $a=b=c=1$, the $M c G L F R$ distribution reduces to GLFR distribution which introduced by Sarhan and Kundu (20).

- $\quad$ Beta Generalized Linear Failure Rate distribution: For $c=1$, the $M c G L F R$ distribution reduces to $B G L F R$ distribution.

- Beta Linear Failure Rate distribution. For $c=\theta=1$, the $M c G L F R$ distribution reduces to BLFR distribution which introduced by Jafari and Mahmoudi (2012).

- Kumaraswamy Generalized Linear Failure Rate distribution: For $a=1$, the $M c G L F R$ distribution reduces to KGLFR distribution which introduced by Elbatal (2013).

- McDonald Generalized Exponential distribution: For $\beta=0$, the McGLFR distribution reduces to $M c G E$ distribution.

- Generalized Exponential distribution: For $\beta=0$, and $a=b=c=1$, the $M c G L F R$ distribution reduces to $G E$ distribution which introduced by Gupta and Kundu (1999).

- McDonald Exponential distribution: For $\beta=0$, and $\theta=1$, the $M c G L F R$ distribution reduces to $M c E$ distribution.

- Beta Exponential distribution: For $\beta=0$, and $\theta=c=1$, the $M c G L F R$ distribution reduces to $B E$ distribution.

- Kumaraswamy Exponential distribution: For $\beta=0$, and $\theta=a=1$, the $M c G L F R$ distribution reduces to $K E$ distribution.

- McDonald Generalized Rayleigh distribution: For $\alpha=0$, the McGLFR distribution reduces to $M c G R$ distribution.

- Generalized Rayleigh distribution: For $\alpha=0$, and $a=b=c=1$, the McGLFR distribution reduces to $G R$ distribution which introduced by Kundu and Rakab (2005).

- McDonald Rayleigh distribution: For $\alpha=0$, and $\theta=1$, the McGLFR distribution reduces to $M c R$ distribution.

\section{Statistical Properties}

In this section we study the statistical properties of the (McGLFR) distribution, specifically moments and moment generating function.Moments are necessary and 
important in any statistical analysis, especially in applications. It can be used to study the most important features and characteristics of a distribution (e.g., tendency, dispersion, skewness and kurtosis).

\section{Theorem 3.1}

The $r$ th moment of $(M c G L F R)$ distribution, $r=1,2, \ldots$ is given by

$$
\begin{aligned}
\mu_{r}^{\prime} & =\frac{c \theta}{B(a, b)} \sum_{i=j=k=0}^{\infty}(-1)^{i+j+k}\left(\begin{array}{c}
b-1 \\
i
\end{array}\right)\left(\begin{array}{c}
\theta c(a+i)-1 \\
j
\end{array}\right) \frac{(\beta(j+1))^{k}}{2^{k} k !} \\
& \times\left[\frac{\alpha \Gamma(r+2 k+1)}{[\alpha(j+1)]^{r+2 k+1}}+\beta \frac{\Gamma(r+2(k+1))}{[\alpha(j+1)]^{r+2(k+1)}}\right]
\end{aligned}
$$

\section{Proof.}

We start with the well known definition of the $r$ th moment of the random variable $X$ with probability density function $f(x)$ given by

$$
\mu_{r}^{\prime}=\int_{0}^{\infty} x^{r} f(x, \varphi) d x
$$

Substituting from (7) into the above relation, we get

$$
\mu_{r}^{\prime}=\frac{c \theta}{B(a, b)} \int_{0}^{\infty} x^{r}(\alpha+\beta x) e^{-\left(\alpha x+\frac{\beta}{2} x^{2}\right)}\left[1-e^{-\left(\alpha x+\frac{\beta}{2} x^{2}\right)}\right]^{\theta a c-1}\left\{1-\left[1-e^{-\left(\alpha x+\frac{\beta}{2} x^{2}\right)}\right]^{\theta c}\right\}^{b-1} d x
$$

since $0<e^{-\left(\alpha x+\frac{\beta}{2} x^{2}\right)}<1$, for $x>0$, the binomial series expansion of

$$
\begin{aligned}
& \left\{1-\left[1-e^{-\left(\alpha x+\frac{\beta}{2} x^{2}\right)}\right]^{\theta c}\right\} \text { yields } \\
& \left\{1-\left[1-e^{-\left(\alpha x+\frac{\beta}{2} x^{2}\right)}\right]^{\theta c}\right\}^{b-1}=\sum_{i=0}^{\infty}(-1)^{i}\left(\begin{array}{c}
b-1 \\
i
\end{array}\right)\left(1-e^{-\left(\alpha x+\frac{\beta}{2} x^{2}\right)}\right)^{\theta i c},
\end{aligned}
$$

thus we get

$$
\mu_{r}^{\prime}=\frac{c \theta}{B(a, b)} \sum_{i=0}^{\infty}(-1)^{i}\left(\begin{array}{c}
b-1 \\
i
\end{array} \int_{0}^{\infty} x^{r}(\alpha+\beta x) e^{-\left(\alpha x+\frac{\beta}{2} x^{2}\right)}\left[1-e^{-\left(\alpha x+\frac{\beta}{2} x^{2}\right)}\right]^{\theta c(a+i)-1} d x\right.
$$

Again, the binomial series expansion of $\left[1-e^{-\left(\alpha x+\frac{\beta}{2} x^{2}\right)}\right]^{\theta c(a+i)-1}$ yields

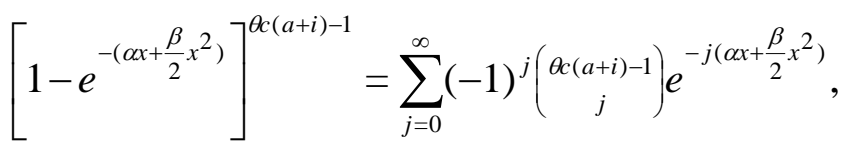

we obtain

$$
\mu_{r}^{\prime}=\frac{c \theta}{B(a, b)} \sum_{i=j=0}^{\infty}(-1)^{i+j}\left(\begin{array}{c}
b-1 \\
i
\end{array}\right)\left(\begin{array}{c}
\theta c(a+i)-1 \\
j
\end{array}\right) \int_{0}^{\infty} x^{r}(\alpha+\beta x) e^{-(j+1)\left(\alpha x+\frac{\beta}{2} x^{2}\right)} d x,
$$


but the series expansion of $e^{-(j+1)\left(\frac{\beta}{2} x^{2}\right)}$ is given by

$$
e^{-(j+1)\left(\frac{\beta}{2} x^{2}\right)}=\sum_{k=0}^{\infty} \frac{(-\beta(j+1))^{k} x^{2 k}}{2^{k} k !},
$$

substituting from (17) into (16), yields

$$
\mu_{r}^{\prime}=C_{i, j, k} \int_{0}^{\infty} x^{r+2 k}(\alpha+\beta x) e^{-\alpha(j+1) x} d x
$$

where

$$
C_{i, j, k}=\frac{c \theta}{B(a, b)} \sum_{i=j=k=0}^{\infty}(-1)^{i+j+k}\left(\begin{array}{c}
b-1 \\
i
\end{array}\right)\left(\begin{array}{c}
\theta c(a+i)-1 \\
j
\end{array}\right) \frac{(\beta(j+1))^{k}}{2^{k} k !} .
$$

the integral in (18) can be computed as follows

$$
\begin{aligned}
\mu_{r}^{\prime} & =C_{i, j, k}\left[\alpha \int_{0}^{\infty} x^{r+2 k} e^{-\alpha(j+1) x} d x+\beta \int_{0}^{\infty} x^{r+2 k+1} e^{-\alpha(j+1) x} d x\right] \\
& =C_{i, j, k}\left[\frac{\alpha \Gamma(r+2 k+1)}{[\alpha(j+1)]^{r+2 k+1}}+\beta \frac{\Gamma(r+2(k+1))}{[\alpha(j+1)]^{r+2(k+1)}}\right] .
\end{aligned}
$$

wich completes the proof.

\section{Theorem 3.2.}

The moment generating function of (McGLFR) distribution is given by

$$
M(t)=C_{i, j, k}\left[\frac{\alpha \Gamma(2 k+1)}{[\alpha(j+1)-t]^{2 k+1}}+\beta \frac{\Gamma(2(k+1))}{[\alpha(j+1)-t]^{2(k+1)}}\right] .
$$

\section{Proof.}

We start with the well known definition of the $M(t)$ of the random variable $X$ with probability density function $f(x)$ given by

$$
M(t)=E\left(e^{t X}\right)=\int_{0}^{\infty} e^{t x} f(x, \varphi) d x
$$

Substituting from (7) into the above relation, we get

$$
M(t)=\frac{c \theta}{B(a, b)} \int_{0}^{\infty} e^{t x}(\alpha+\beta x) e^{-\left(\alpha x+\frac{\beta}{2} x^{2}\right)}\left[1-e^{-\left(\alpha x+\frac{\beta}{2} x^{2}\right)}\right]^{\theta a c-1}\left\{1-\left[1-e^{-\left(\alpha x+\frac{\beta}{2} x^{2}\right)}\right]^{\theta c}\right\}^{b-1} d x
$$

using the binomial series expansion given by (15) and (17) we get

$$
\begin{aligned}
M(t) & =C_{i, j, k} \int_{0}^{\infty} x^{2 k}(\alpha+\beta x) e^{-x[\alpha(j+1)-t]} d x \\
& =C_{i, j, k}\left[\alpha \int_{0}^{\infty} x^{2 k} e^{-x[\alpha(j+1)-t]} d x+\beta \int_{0}^{\infty} x^{2 k+1} e^{-x[\alpha(j+1)-t]} d x\right] \\
& =C_{i, j, k}\left[\frac{\alpha \Gamma(2 k+1)}{[\alpha(j+1)-t]^{2 k+1}}+\beta \frac{\Gamma(2(k+1))}{[\alpha(j+1)-t]^{2(k+1)}}\right] .
\end{aligned}
$$

which completes the proof. 


\subsection{Conditional moments}

For lifetime models, it is also of interest to find the conditional moments and the mean residual liftime function. The conditional moments for $(M c G L F R)$ distribution is given by

$$
\begin{aligned}
& E\left(X^{n} \mid X>t\right)=\int_{t}^{\infty} x^{n} f(x, \varphi) d x \\
& =\frac{c \theta}{B(a, b)} \int_{t}^{\infty} x^{n}(\alpha+\beta x) e^{-\left(\alpha x+\frac{\beta}{2} x^{2}\right)}\left[1-e^{-\left(\alpha x+\frac{\beta}{2} x^{2}\right)}\right]^{\theta a c-1}\left\{1-\left[1-e^{-\left(\alpha x+\frac{\beta}{2} x^{2}\right)}\right]^{\theta c}\right\}^{b-1} d x
\end{aligned}
$$

using (14), (15) and (17), Equation (24) becomes

$$
\begin{aligned}
E\left(X^{n} \mid X>t\right) & =C_{i, j, k} \int_{t}^{\infty} x^{2 k+n}(\alpha+\beta x) e^{-\alpha(j+1) x} d x \\
& =C_{i, j, k}\left[\frac{\alpha \Gamma(2 k+n+1, \alpha(j+1) t)}{[\alpha(j+1)]^{2 k+n+1}}+\frac{\beta \Gamma(2 k+n+2, \alpha(j+1) t)}{[\alpha(j+1)]^{2 k+n+2}}\right]
\end{aligned}
$$

The mean residual lifetime function is given by

$$
E(X \mid X>t)-t=C_{i, j, k}\left[\frac{\alpha \Gamma(2 k+2, \alpha(j+1) t)}{[\alpha(j+1)]^{2 k+2}}+\frac{\beta \Gamma(2 k+3, \alpha(j+1) t)}{[\alpha(j+1)]^{2 k+3}}\right]-t
$$

\subsection{Mean deviation}

The amount of scatter in a population is evidently measured to some extent by the totality of deviations from the mean and median. These are known as the mean deviation about the mean and the mean deviation about the median are defined by

$$
\delta_{1}(x)=\int_{0}^{\infty}|x-\mu| f(x) d x
$$

and

$$
\delta_{2}(x)=\int_{0}^{\infty}|x-M| f(x) d x
$$

respectively, where $\mu=E(X)$ and $\mathrm{M}=\operatorname{Median}(\mathrm{X})$ denotes the median. The measures $\delta_{1}(x)$ and $\delta_{2}(x)$ can be calculated using the relationships

$$
\begin{aligned}
\delta_{1}(x) & =\int_{0}^{\infty}|x-\mu| f(x) d x \\
& =\int_{0}^{\mu}(\mu-x) f(x) d x+\int_{\mu}^{\infty}(x-\mu) f(x) d x \\
& =\mu F(\mu)-\int_{0}^{\mu} x f(x) d x-\mu[1-F(\mu)]+\int_{\mu}^{\infty} x f(x) d x \\
& =2 \mu F(\mu)-2 \mu+2 \int_{\mu}^{\infty} x f(x) d x .
\end{aligned}
$$


Also

$$
\delta_{2}(x)=\int_{0}^{\infty}|x-M| f(x) d x=-\mu+2 \int_{M}^{\infty} x f(x) d x .
$$

we can calculate Equations (26) and (27) as follows, from equation (12), when $r=1$ we get

and

$$
\mu=C_{i, j, k}\left[\frac{\alpha \Gamma(2 k+2)}{[\alpha(j+1)]^{2 k+2}}+\beta \frac{\Gamma(2 k+3)}{[\alpha(j+1)]^{2 k+3}}\right]
$$

$$
\int_{\mu}^{\infty} x f(x) d x=\frac{c \theta}{B(a, b)} \int_{\mu}^{\infty} x(\alpha+\beta x) e^{-\left(\alpha x+\frac{\beta}{2} x^{2}\right)}\left[1-e^{-\left(\alpha x+\frac{\beta}{2} x^{2}\right)}\right]^{\theta a c-1}\left\{1-\left[1-e^{-\left(\alpha x+\frac{\beta}{2} x^{2}\right)}\right]^{\theta c}\right\}^{b-1} d x
$$

using (14), (15) and (17), the formula above imply

$$
\begin{aligned}
\int_{\mu}^{\infty} x f(x) d x & =C_{i, j, k} \int_{\mu}^{\infty} x^{2 k+1}(\alpha+\beta x) e^{-\alpha(j+1) x} d x \\
& =C_{i, j, k}\left[\frac{\alpha \Gamma(2 k+2, \alpha(j+1) \mu)}{[\alpha(j+1)]^{2 k+2}}+\frac{\beta \Gamma(2 k+3, \alpha(j+1) \mu)}{[\alpha(j+1)]^{2 k+3}}\right] .
\end{aligned}
$$

and

$$
\int_{M}^{\infty} x f(x) d x=C_{i, j, k}\left[\frac{\alpha \Gamma(2 k+2, \alpha(j+1) M)}{[\alpha(j+1)]^{2 k+2}}+\frac{\beta \Gamma(2 k+3, \alpha(j+1) M)}{[\alpha(j+1)]^{2 k+3}}\right] .
$$

so that

$$
\delta_{1}(x)=2 \mu F(\mu)-2 \mu+2 C_{i, j, k}\left[\frac{\alpha \Gamma(2 k+2, \alpha(j+1) \mu)}{[\alpha(j+1)]^{2 k+2}}+\frac{\beta \Gamma(2 k+3, \alpha(j+1) \mu)}{[\alpha(j+1)]^{2 k+3}}\right] .
$$

and

$$
\delta_{2}(x)=-\mu+2 C_{i, j, k}\left[\frac{\alpha \Gamma(2 k+2, \alpha(j+1) M)}{[\alpha(j+1)]^{2 k+2}}+\frac{\beta \Gamma(2 k+3, \alpha(j+1) M)}{[\alpha(j+1)]^{2 k+3}}\right] .
$$

where $\Gamma(s, b)$ is the upper incomplete gamma function given by

$$
\Gamma(s, b)=\int_{b}^{\infty} t^{s-1} e^{-t} d t
$$

\subsection{Bonferroni and Lorenz Curves}

In this section we proposed the Bonferroni and Lorenz Curves. The Bonferroni and Lorenz curves (Bonferroni 1930) and the Bonferroni and Gini indices have applications not only in economics to study income and poverty, but also in other fields like reliability, demography, insurance and medicine. The Bonferroni and Lorenz curves are defined by

$$
\begin{aligned}
B(p) & =\frac{1}{p \mu} \int_{0}^{q} x f(x) d x \\
& =\frac{1}{p \mu} C_{i, j, k}\left[\frac{\alpha \gamma(2 k+2, \alpha(j+1) q)}{[\alpha(j+1)]^{2 k+2}}+\frac{\beta \gamma(2 k+3, \alpha(j+1) q)}{[\alpha(j+1)]^{2 k+3}}\right]
\end{aligned}
$$


and

$$
\begin{aligned}
L(p) & =\frac{1}{\mu} \int_{0}^{q} x f(x) d x \\
& =\frac{1}{\mu} C_{i, j, k}\left[\frac{\alpha \gamma(2 k+2, \alpha(j+1) q)}{[\alpha(j+1)]^{2 k+2}}+\frac{\beta \gamma(2 k+3, \alpha(j+1) q)}{[\alpha(j+1)]^{2 k+3}}\right] .
\end{aligned}
$$

where $\gamma(\alpha, b)$ is the lower incomplete gamma function given by

$$
\gamma(\alpha, b)=\int_{0}^{b} t^{\alpha-1} e^{-t} d t
$$

\section{Distribution of the order statistics}

In this section, we derive closed form expressions for the pdfs of the $r_{t h}$ order statistic of the $(M c G L F R)$ distribution, also, the measures of skewness and kurtosis of the distribution of the $r_{t h}$ order statistic in a sample of size $n$ for different choices of $n ; r$ are presented in this section. Let $X_{1}, X_{2}, \ldots, X_{n}$ be a simple random sample from (McGLFR) distribution with pdf and cdf given by (7) and (9), respectively. Let $X_{1} \leq X_{2} \leq, \ldots, \leq X_{n}$ denote the order statistics obtained from this sample. We now give the probability density function of $X_{r: n}$, say $f_{r: n}(x, \varphi)$ and the moments of $X_{r: n}, r=1,2, \ldots, n$. Therefore, the measures of skewness and kurtosis of the distribution of the $X_{r: n}$ are presented. The probability density function of $X_{r: n}$ is given by

$$
f_{r: n}(x, \varphi)=\frac{1}{B(r, n-r+1)}[F(x, \varphi)]^{r-1}[1-F(x, \varphi)]^{n-r} f(x, \varphi)
$$

where $F(x, \Phi)$ and $f(x, \Phi)$ are the cdf and pdf of the $(M c G L F R)$ distribution given by (7), (??), respectively, and $B$ (.,.) is the beta function, since $0<F(x, \varphi)<1$, for $x>0$, by using the binomial series expansion of $[1-F(x, \varphi)]^{n-r}$, given by

$$
[1-F(x, \Phi)]^{n-r}=\sum_{j=0}^{n-r}(-1)^{j}\left(\begin{array}{c}
n-r \\
j
\end{array}\right)[F(x, \varphi)]^{j},
$$

we have

$$
f_{r: n}(x, \varphi)=\sum_{j=0}^{n-r}(-1)^{j}\left(\begin{array}{c}
n-r \\
j
\end{array}\right)[F(x, \varphi)]^{r+j-1} f(x, \varphi),
$$

substituting from (7) and (8) into (32), we can express both $f_{r: n}(x, \varphi)$ in terms of the incomplete beta function and the $k_{t h}$ ordinary moment of the $r_{t h}$ order statistics $X_{r: n}$ say $E\left(X_{r: n}^{k}\right)$ as a liner combination of the $k_{t h}$ moments of the $(M c G L F R)$ distribution with different shape parameters. Therefore, the measures of skewness and kurtosis of the distribution of $X_{r: n}$ can be calculated. 


\section{Estimation and Inference}

In this section we determine the maximum likelihood estimates (MLEs) of the parameters of the $\operatorname{Mc} G \operatorname{LFR}(x, \varphi)$ distribution from complete samples only. Let $X_{1}, X_{2}, \ldots, X_{n}$ be a random sample from $X \sim \operatorname{Mc} \operatorname{GLFR}(x, \varphi)$ with observed values $x_{1}, x_{2}, \ldots, x_{n}$ and let $\Psi=(\alpha, \beta, \theta, a, b, c)^{T}$ be the vector of the model parameters. The log likelihood function of (7) is defined as

$$
\begin{aligned}
L(\varphi)= & n \log c+n \log \theta+n \log [\Gamma(a+b)]-n \log [\Gamma(a)]-n \log [\Gamma(b)] \\
& +\sum_{i=1}^{n} \log \left(\alpha+\beta x_{i}\right)-\alpha \sum_{i=1}^{n} x_{i}-\frac{\beta}{2} \sum_{i=1}^{n} x_{i}^{2} \\
& +(\theta a c-1) \sum_{i=1}^{n} \log \left[1-e^{-\left(\alpha x+\frac{\beta}{2} x^{2}\right)}\right]+(b-1) \sum_{i=1}^{n} \log \left[1-\left(1-e^{-\left(\alpha x+\frac{\beta}{2} x^{2}\right)}\right)^{\theta c}\right],
\end{aligned}
$$

Differentiating $L(\varphi)$ with respect to each parameter $\alpha, \beta, \theta, a, b$ and $c$ and setting the result equals to zero, we obtain maximum likelihood estimates. The partial derivatives of $L(\varphi)$ with respect to each parameter or the score function is given by:

$$
U_{n}(\varphi)=\left(\frac{\partial(L)}{\partial \alpha}, \frac{\partial(L)}{\partial \beta}, \frac{\partial(L)}{\partial \theta}, \frac{\partial(L)}{\partial a}, \frac{\partial(L)}{\partial b}, \frac{\partial(L)}{\partial c}\right),
$$

where

$$
\begin{aligned}
& \frac{\partial(L)}{\partial \alpha}=\sum_{i=1}^{n} \frac{1}{\left(\alpha+\beta x_{i}\right)}-\sum_{i=1}^{n} x_{i}+(\theta a c-1) \sum_{i=1}^{n} \frac{x_{i} e^{-\left(\alpha x+\frac{\beta}{2} x^{2}\right)}}{1-e^{-\left(\alpha x+\frac{\beta}{2} x^{2}\right)}} \\
& -\theta c(b-1) \sum_{i=1}^{n} \frac{x_{i}\left(1-e^{-\left(\alpha x+\frac{\beta}{2} x^{2}\right)}\right)^{\theta c-1} e^{-\left(\alpha x+\frac{\beta}{2} x^{2}\right)}}{\left[1-\left(1-e^{-\left(\alpha x+\frac{\beta}{2} x^{2}\right)}\right)^{\theta c}\right]}=0 \\
& \frac{\partial(L)}{\partial \beta}=\sum_{i=1}^{n} \frac{x_{i}}{\left(\alpha+\beta x_{i}\right)}-\frac{1}{2} \sum_{i=1}^{n} x_{i}^{2} \\
& +(\theta a c-1) \sum_{i=1}^{n} \frac{x_{i}^{2} e^{-\left(\alpha x+\frac{\beta}{2} x^{2}\right)}}{2\left[1-e^{-\left(\alpha x+\frac{\beta}{2} x^{2}\right)}\right]} \\
& +\theta c(b-1) \sum_{i=1}^{n} \frac{x_{i}^{2}\left(1-e^{-\left(\alpha x+\frac{\beta}{2} x^{2}\right)}\right)^{\theta c-1} e^{-\left(\alpha x+\frac{\beta}{2} x^{2}\right)}}{2\left[1-\left(1-e^{-\left(\alpha x+\frac{\beta}{2} x^{2}\right)}\right)^{\theta c}\right]}=0 \\
& \frac{\partial(L)}{\partial \theta}=\frac{n}{\theta}+a c \sum_{i=1}^{n} \log \left[1-e^{-\left(\alpha x+\frac{\beta}{2} x^{2}\right)}\right]+c(b-1) \sum_{i=1}^{n} \frac{\left(1-e^{-\left(\alpha x+\frac{\beta}{2} x^{2}\right)}\right)^{\theta c} \log \left(1-e^{-\left(\alpha x+\frac{\beta}{2} x^{2}\right)}\right)}{\left[1-\left(1-e^{-\left(\alpha x+\frac{\beta}{2} x^{2}\right)}\right)^{\theta c}\right]}=0,
\end{aligned}
$$




$$
\begin{aligned}
& \frac{\partial(L)}{\partial a}=n \psi(a+b)-n \psi(a)+\theta c \sum_{i=1}^{n} \log \left[1-e^{-\left(\alpha x+\frac{\beta}{2} x^{2}\right)}\right]=0, \\
& \frac{\partial(L)}{\partial b}=n \psi(a+b)-n \psi(b)+\sum_{i=1}^{n} \log \left[1-\left(1-e^{-\left(\alpha x+\frac{\beta}{2} x^{2}\right)}\right)^{\theta c}\right]=0,
\end{aligned}
$$

and

$$
\frac{\partial(L)}{\partial c}=\frac{n}{c}+\theta a \sum_{i=1}^{n} \log \left[1-e^{-\left(\alpha x+\frac{\beta}{2} x^{2}\right)}\right]+\theta(b-1) \sum_{i=1}^{n} \frac{\left(1-e^{-\left(\alpha x+\frac{\beta}{2} x^{2}\right)}\right)^{\theta c} \log \left(1-e^{-\left(\alpha x+\frac{\beta}{2} x^{2}\right)}\right)}{\left[1-\left(1-e^{-\left(\alpha x+\frac{\beta}{2} x^{2}\right)}\right)^{\theta c}\right]}=0,
$$

where $\psi($.$) is digamma function \psi(x)=\frac{d}{d x} \log \Gamma(x)=\frac{\Gamma^{\prime}(x)}{\Gamma(x)}$, and the MLE of the parameters $\alpha, \beta, \theta, a, b$, and $c$, say $\alpha, \beta, \vec{\theta}, \vec{a}, \vec{b}$, and $\stackrel{\square}{c}$ are obtained by solving the following equations, $\frac{\partial(L)}{\partial \alpha}=\frac{\partial(L)}{\partial \beta}=\frac{\partial(L)}{\partial \theta}=\frac{\partial(L)}{\partial a}=\frac{\partial(L)}{\partial b}=\frac{\partial(L)}{\partial c}=0$. There is no closed form solution to these equations, so numerical technique must be applied.

These non-linear can be routinely solved using Newton's method or fixed point iteration techniques. The subroutines to solve non-linear optimization problem are available in $\mathrm{R}$ software namely optim(), nlm() and bbmle() etc. We used nlm() package.

\section{Application}

Now we use a real data set to show that the McGLFR distribution can be a better model than the GLFR distribution.

We work with nicotine measurements made from several brands of cigarettes in 1998. The data have been collected by the Federal Trade Commission which is an independent agency of the US government, whose main mission is the promotion of consumer protection.

The report entitled tar, nicotine, and carbon monoxide of the smoke of 1206 varieties of domestic cigarettes for the year of 1998 available at http://www.ftc.gov/reports/tobacco and consists of the data sets and some information about the source of the data, smoker's behaviour and beliefs about nicotine, tar and carbon monoxide contents in cigarettes. The free form data set can be found at http://pw1.netcom.com/ rdavis2/smoke.html.

The site http://home.att.net/ rdavis2/cigra.html contains $n=384$ observations. We analysed data on nicotine, measured in milligrams per cigarette, from several cigarette brands. Some summary statistics for the nicotine data are as follows: mean $=0.852$, median $=0.9$, minimum $=0.1$ and maximum $=2$. 
Table 1: Estimated parameters of the McGLFR, GLFR, Rayleigh and Transmuted Rayleigh distribution for the nicotine measurements data.

\begin{tabular}{|c|c|c|}
\hline Model & $\begin{array}{c}\text { Parameter } \\
\text { Estimate }\end{array}$ & $-\ell(\cdot ; x)$ \\
\hline \multirow{4}{*}{ McGLFR } & $\hat{a}=0.571$ & \multirow{2}{*}{110.970} \\
\cline { 2 - 2 } & $\hat{b}=6.259$ & \multirow{4}{*}{} \\
\cline { 2 - 2 } & $\hat{c}=2.234$ & \\
\cline { 2 - 2 } & $\hat{\theta}=1.369$ & \\
\cline { 2 - 2 } & $\hat{\alpha}=0.195$ & \multirow{2}{*}{117.575} \\
\cline { 2 - 2 } & $\hat{\beta}=0.826$ & \\
\hline GLFR & $\hat{\theta}=2.029$ & \multirow{2}{*}{121.224} \\
\cline { 2 - 2 } & $\hat{\alpha}=0.325$ & \\
\cline { 2 - 2 } & $\hat{\beta}=2.921$ & 142.357 \\
\hline Transmuted & $\hat{\sigma}=0.555$ & \\
\cline { 2 - 2 } Rayleigh & $\hat{\lambda}=-0.771$ & \\
\hline Rayleigh & $\hat{\sigma}=0.647$ & \\
\hline
\end{tabular}

The LR test statistic to test the hypotheses $H_{0}: a=b=c=1$ versus $H_{1}: a \neq 1 \vee b \neq 1 \vee c \neq 1$ is $\omega=13.21>7.815=\chi_{3 ; 0.05}^{2}$, so we reject the null hypothesis.

\section{Ecdf of distances}

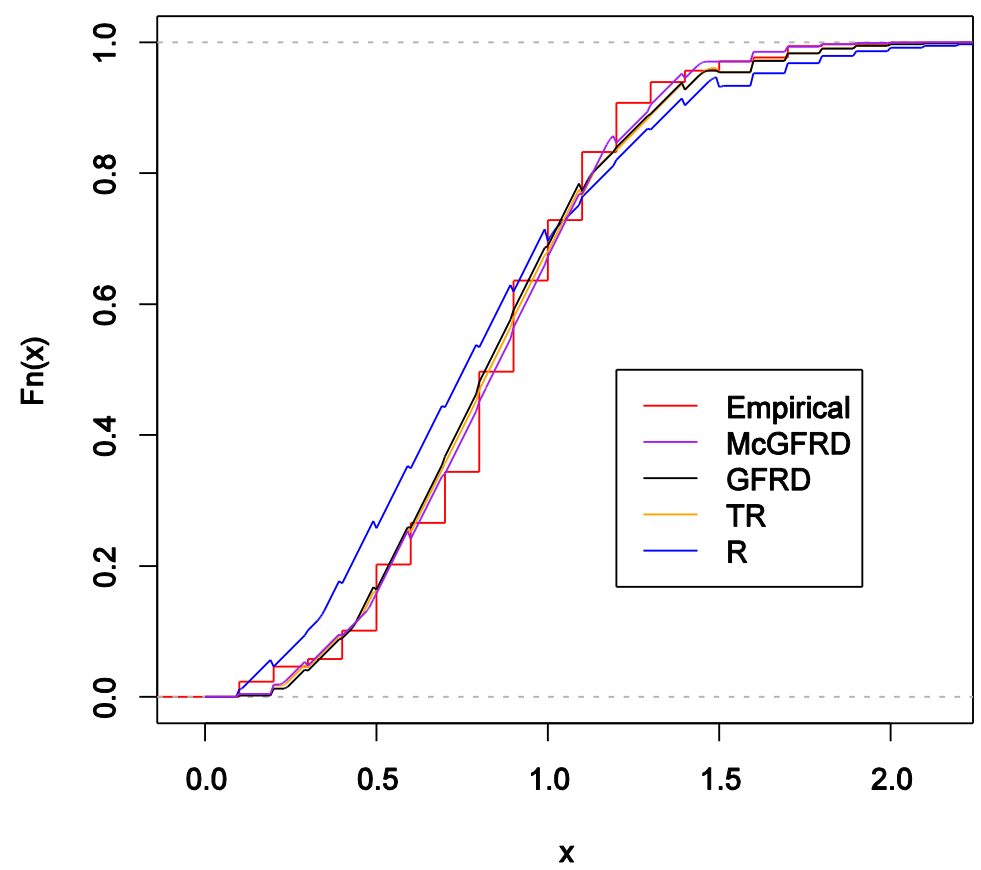

Figure 5: Empirical, fitted McGLFR, GLFR, Rayleigh, and transmuted Rayleigh cdf of the nicotine measurements data. 
Table 2: Criteria for comparison.

\begin{tabular}{|c|c|c|c|c|}
\hline Model & KS & $-2 \ell$ & AIC & AICC \\
\hline McGLFR & 0.104 & 221.94 & 233.94 & 234.187 \\
\hline GLFR & 0.114 & 235.15 & 241.15 & 241.220 \\
\hline TRayleigh & 0.124 & 242.448 & 246.448 & 243.445 \\
\hline Rayleigh & 0.184 & 284.714 & 286.714 & 285.714 \\
\hline
\end{tabular}

In order to compare the two distribution models, we consider criteria like KS (Kolmogorow Smirnow), $-2 \ell$, AIC (Akaike information criterion), and AICC (corrected Akaike information criterion), for the data set. The better distribution corresponds to smaller $\mathrm{KS},-2 \ell, \mathrm{AIC}$ and AICC values:

$$
A I C=2 k-2 \ell, \quad A I C C=A I C+\frac{2 k(k+1)}{n-k-1},
$$

where $k$ is the number of parameters in the statistical model, $n$ the sample size and $\ell$ is the maximized value of the log-likelihood function under the considered model. Table 1 shows the MLEs under both distributions, Table 2 shows the values of KS, $-2 \ell$, AIC, and AICC, values. The values in Table 2 indicate that the McGLFR distribution leads to a better fit than the GLFR distribution.

\section{Conclusion}

Here we propose a new model, the so-called the McGLFR distribution which extends the GLFR distribution in the analysis of data with real support. An obvious reason for generalizing a standard distribution is because the generalized form provides larger flexibility in modeling real data. We derive expansions for the moments and for the moment generating function. The estimation of parameters is approached by the method of maximum likelihood, also the information matrix is derived. We consider the likelihood ratio statistic to compare the model with its baseline model. An application of the McGLFR distribution to real data show that the new distribution can be used quite effectively to provide better fits than the GLFR distribution.

\section{References}

1. Cordeiro G. M. and de Castro, M. (2011). A new family of generalized distributions. Journal of Statistical Computation and Simulation, 81, 883-898.

2. Cordeiro, G.M., Cintra, R. J , Rêgo, L.C and Ortega, E.M (2012). The McDonald Normal Distribution. Pakistan Journal of Statistics and Operation Rersearch, 8(3), 301-329.

3. Cordeiro, G.M., Elizabeth M. H and Ortega, E. M (2012). The McDonald Weibull model. Statistics, 1-23.

4. Elbatal. I (2013). Kumaraswamy Generalized linear failure rate. Indian Journal of Computational and Applied Mathematics, 1(1), 61-78.

5. Eugene, N., Lee, C. and Famoye, F. (2002). Beta-normal distribution and its applications. Communications in Statistics: Theory and Methods, 31, 497-512. 
6. Francisco W. P. Nascimento, A. D, Santos-Neto, M and Cordeiro, G. M (2012). The Mc- $\Gamma$ Distribution and Its Statistical Properties: An Application to Reliability Data. International Journal of Statistics and Probability, 1(1), 53-71.

7. Gradshteyn, I.S., and Ryzhik, I.M. (2000). Table of Integrals, Series, and Products. Academic Press, New York. Transactions on Reliability. 42:299-302.

8. Gupta, R. D., Kundu, D. (1999). Generalized exponential distribution. Australian and New Zealand Journal of Statistics, 41 (2), 173-188.

9. Haupt, E, Schabe, H. (1992). A new model for a lifetime distribution with bathtub shaped failure rate. Microelectronics and Reliability, 32, 633639.

10. Hjorth, U. (1980). A reliability distribution with increasing, decreasing, constant and bathtub failure rates. Technometrics, 22, 99107.

11. Jafari. A and Mahmoudi. E (2012) . Beta-Linear Failure Rate Distribution and its Applications. arXiv:1212.5615v1 [stat.ME].

12. Johnson, N. L., Kotz, S and N. Balakrishnan (1995). Continuous Univariate Distribution, V(2), 2nd edition, New York, Wiley.

13. Kundu, D, Rakab, M.Z, (2005). Generalized Rayleigh distribution: Different methods of estimation. Computational Statistics and Data Analysis, 49, 187200.

14. Lawless, J.F. (2003). Statiatical Models and Methods for Lifetime Data. John Wiely and Sons, New York.

15. Lai, C.D, Xie, M, Murthy, D.N.P. (2001). Bathtub shaped failure rate distributions, in Handbook in Reliability, N. Balakrishnan and C. R. Rao, Eds., 20, 69 - 104.

16. Merovci, F. (2013). Transmuted Rayleigh distribution. Austrian Journal of Statistics, 42(1), 21-31.

17. Nelson, W. (1982). Lifetime Data Analysis. Wiley, New York.

18. Oluyede and Rajasooriya (2013). The McDagum distribution and its Statistical Properties with Applications . Asian Journal oF Mathematics and Applications.

19. Rajarshi, S., Rajarshi, M.B. (1988). Bathtub distributions: A review. Communications in Statistics-Theory and Methods, 17, 25212597.

20. Sarhan, A. M. and Kundu, D. (2009). Generalized linear failure rate distribution. Communications in Statistics-Theory and Methods, 38, 642-660.

21. Swain. J, S. Venkatraman. S, and Wilson. J. (1988). Least squares estimation of distribution function in Johnson's translation system. Journal of Statistical Computation and Simulation, 29, 271 - 297. 
McDonald Generalized Linear Failure Rate Distribution

Appendix

The elements of Hessian matrix:

$$
\begin{aligned}
& V_{\alpha \alpha}=-\sum_{i=1}^{n}\left(\alpha+\beta x_{i}\right)^{-2}-(\theta a c-1) \sum_{i=1}^{n} \frac{x_{i}^{2} \mathrm{e}^{-1 / 2 x_{i}\left(\beta x_{i}+2 \alpha\right)}}{\left(-1+\mathrm{e}^{-1 / 2 x_{i}\left(\beta x_{i}+2 \alpha\right)}\right)^{2}} \\
& -(b-1) \sum_{i=1}^{n} \frac{\theta c x_{i}^{2} \mathrm{e}^{-1 / 2 x_{i}\left(\beta x_{i}+2 \alpha\right)} A_{1}}{\left(-1+\mathrm{e}^{-1 / 2 x_{i}\left(\beta x_{i}+2 \alpha\right)}\right)^{2}\left(-1+\left(\left(\mathrm{e}^{\alpha x_{i}}-\mathrm{e}^{-1 / 2 \beta x_{i}^{2}}\right) \mathrm{e}^{-\alpha x_{i}}\right)^{\theta c}\right)^{2}} \\
& A_{1}=\left(\left(\mathrm{e}^{\alpha x_{i}}-\mathrm{e}^{-1 / 2 \beta x_{i}{ }^{2}}\right) \mathrm{e}^{-\alpha x_{i}}\right)^{\theta c} \theta c \mathrm{e}^{-\alpha x_{i}-1 / 2 \beta x_{i}{ }^{2}}-\left(\left(\mathrm{e}^{\alpha x_{i}}-\mathrm{e}^{-1 / 2 \beta x_{i}{ }^{2}}\right) \mathrm{e}^{-\alpha x_{i}}\right)^{\theta c} \\
& +\left(\left(\mathrm{e}^{\alpha x_{i}}-\mathrm{e}^{-1 / 2 \beta x_{i}^{2}}\right) \mathrm{e}^{-\alpha x_{i}}\right)^{2 \theta c} \\
& V_{\alpha \beta}=-\sum_{i=1}^{n} \frac{x_{i}}{\left(\alpha+\beta x_{i}\right)^{2}}-\frac{(\theta a c-1)}{2} \sum_{i=1}^{n} \frac{x_{i}^{3} \mathrm{e}^{-1 / 2 x_{i}\left(\beta x_{i}+2 \alpha\right)}}{\left(-1+\mathrm{e}^{-1 / 2 x_{i}\left(\beta x_{i}+2 \alpha\right)}\right)^{2}} \\
& -1 / 2 \sum_{i=1}^{n} \frac{\theta c x_{i}^{3} \mathrm{e}^{-1 / 2 x_{i}\left(\beta x_{i}+2 \alpha\right)} D_{1}}{\left(-1+\mathrm{e}^{-1 / 2 x_{i}\left(\beta x_{i}+2 \alpha\right)}\right)^{2}\left(-1+\left(\left(\mathrm{e}^{\alpha x_{i}}-\mathrm{e}^{-1 / 2 \beta x_{i}^{2}}\right) \mathrm{e}^{-\alpha x_{i}}\right)^{\theta c}\right)^{2}} \\
& V_{\alpha \theta}=a c \sum_{i=1}^{n} \frac{x_{i} \mathrm{e}^{-\alpha x_{i}-1 / 2 \beta x_{i}^{2}}}{1-\mathrm{e}^{-\alpha x_{i}-1 / 2 \beta x_{i}^{2}}} \\
& D_{1}=\left(\left(\mathrm{e}^{\alpha x_{i}}-\mathrm{e}^{-1 / 2 \beta x_{i}{ }^{2}}\right) \mathrm{e}^{-\alpha x_{i}}\right)^{\theta c} \theta c \mathrm{e}^{-\alpha x_{i}-1 / 2 \beta x_{i}{ }^{2}}-\left(\left(\mathrm{e}^{\alpha x_{i}}-\mathrm{e}^{-1 / 2 \beta x_{i}{ }^{2}}\right) \mathrm{e}^{-\alpha x_{i}}\right)^{\theta c} \\
& +\left(\left(\mathrm{e}^{\alpha x_{i}}-\mathrm{e}^{-1 / 2 \beta x_{i}^{2}}\right) \mathrm{e}^{-\alpha x_{i}}\right)^{2 \theta c} \\
& +(b-1) \sum_{i=1}^{n} \frac{c x_{i} \mathrm{e}^{-1 / 2 x_{i}\left(\beta x_{i}+2 \alpha\right)} A_{2}}{\left(-1+\mathrm{e}^{-1 / 2 x_{i}\left(\beta x_{i}+2 \alpha\right)}\right)\left(-1+\left(\left(\mathrm{e}^{\alpha x_{i}}-\mathrm{e}^{-1 / 2 \beta x_{i}^{2}}\right) \mathrm{e}^{-\alpha x_{i}}\right)^{\theta c}\right)^{2}} \\
& A_{2}=\left(\left(\mathrm{e}^{\alpha x_{i}}-\mathrm{e}^{-1 / 2 \beta x_{i}^{2}}\right) \mathrm{e}^{-\alpha x_{i}}\right)^{\theta c} c \ln \left(1-\mathrm{e}^{-1 / 2 x_{i}\left(\beta x_{i}+2 \alpha\right)}\right) \theta \\
& +\left(\left(\mathrm{e}^{\alpha x_{i}}-\mathrm{e}^{-1 / 2 \beta x_{i}^{2}}\right) \mathrm{e}^{-\alpha x_{i}}\right)^{\theta c}-\left(\left(\mathrm{e}^{\alpha x_{i}}-\mathrm{e}^{-1 / 2 \beta x_{i}^{2}}\right) \mathrm{e}^{-\alpha x_{i}}\right)^{2 \theta c}
\end{aligned}
$$

Pak.j.stat.oper.res. Vol.X No.3 2014 pp267-288

285 
Ibrahim Elbatal, Faton Merovci, W. Marzouk

$$
\begin{aligned}
& V_{\alpha a}=\theta c \sum_{i=1}^{n} \frac{x_{i} \mathrm{e}^{-\alpha x_{i}-1 / 2 \beta x_{i}^{2}}}{1-\mathrm{e}^{-\alpha x_{i}-1 / 2 \beta x_{i}^{2}}} \\
& V_{\alpha b}=\theta c \sum_{i=1}^{n} \frac{\left(1-\mathrm{e}^{-1 / 2 x_{i}\left(\beta x_{i}+2 \alpha\right)}\right)^{\theta c-1} x_{i} \mathrm{e}^{-1 / 2 x_{i}\left(\beta x_{i}+2 \alpha\right)}}{-1+\left(1-\mathrm{e}^{-1 / 2 x_{i}\left(\beta x_{i}+2 \alpha\right)}\right)^{\theta c}} \\
& V_{\alpha c}=\theta a \sum_{i=1}^{n} \frac{x_{i} \mathrm{e}^{-\alpha x_{i}-1 / 2 \beta x_{i}^{2}}}{1-\mathrm{e}^{-\alpha x_{i}-1 / 2 \beta x_{i}^{2}}} \\
& +(b-1) \sum_{i=1}^{n} \frac{\theta x_{i} \mathrm{e}^{-1 / 2 x_{i}\left(\beta x_{i}+2 \alpha\right)} A_{3}}{\left(-1+\mathrm{e}^{-1 / 2 x_{i}\left(\beta x_{i}+2 \alpha\right)}\right)\left(-1+\left(\left(\mathrm{e}^{\alpha x_{i}}-\mathrm{e}^{-1 / 2 \beta x_{i}^{2}}\right) \mathrm{e}^{-\alpha x_{i}}\right)^{\theta c}\right)^{2}} \\
& A_{3}=\left(\left(\mathrm{e}^{\alpha x_{i}}-\mathrm{e}^{-1 / 2 \beta x_{i}^{2}}\right) \mathrm{e}^{-\alpha x_{i}}\right)^{\theta c} c \ln \left(1-\mathrm{e}^{-1 / 2 x_{i}\left(\beta x_{i}+2 \alpha\right)}\right) \theta \\
& +\left(\left(\mathrm{e}^{\alpha x_{i}}-\mathrm{e}^{-1 / 2 \beta x_{i}^{2}}\right) \mathrm{e}^{-\alpha x_{i}}\right)^{\theta c}-\left(\left(\mathrm{e}^{\alpha x_{i}}-\mathrm{e}^{-1 / 2 \beta x_{i}^{2}}\right) \mathrm{e}^{-\alpha x_{i}}\right)^{2 \theta c} \\
& V_{\beta \beta}=-\sum_{i=1}^{n} \frac{x_{i}^{2}}{\left(\alpha+\beta x_{i}\right)^{2}}-\frac{(\theta a c-1)}{4} \sum_{i=1}^{n} \frac{x_{i}^{4} \mathrm{e}^{-1 / 2 x_{i}\left(\beta x_{i}+2 \alpha\right)}}{\left(-1+\mathrm{e}^{-1 / 2 x_{i}\left(\beta x_{i}+2 \alpha\right)}\right)^{2}} \\
& -\frac{(b-1)}{4} \sum_{i=1}^{n} \frac{\theta c x_{i}^{4} \mathrm{e}^{-1 / 2 x_{i}\left(\beta x_{i}+2 \alpha\right)} B_{1}}{\left(-1+\mathrm{e}^{-1 / 2 x_{i}\left(\beta x_{i}+2 \alpha\right)}\right)^{2}\left(-1+\left(\left(\mathrm{e}^{\alpha x_{i}}-\mathrm{e}^{-1 / 2 \beta x_{i}^{2}}\right) \mathrm{e}^{-\alpha x_{i}}\right)^{\theta c}\right)^{2}} \\
& B_{1}=\left(\left(\mathrm{e}^{\alpha x_{i}}-\mathrm{e}^{-1 / 2 \beta x_{i}^{2}}\right) \mathrm{e}^{-\alpha x_{i}}\right)^{\theta c} \theta c \mathrm{e}^{-\alpha x_{i}-1 / 2 \beta x_{i}^{2}}-\left(\left(\mathrm{e}^{\alpha x_{i}}-\mathrm{e}^{-1 / 2 \beta x_{i}^{2}}\right) \mathrm{e}^{-\alpha x_{i}}\right)^{\theta c} \\
& +\left(\left(\mathrm{e}^{\alpha x_{i}}-\mathrm{e}^{-1 / 2 \beta x_{i}^{2}}\right) \mathrm{e}^{-\alpha x_{i}}\right)^{2 \theta c} \\
& V_{\beta \theta}=\frac{a c}{2} \sum_{i=1}^{n} 1 / 2 \frac{x_{i}^{2} \mathrm{e}^{-\alpha x_{i}-\beta x_{i}^{2}}}{1-\mathrm{e}^{-\alpha x_{i}-1 / 2 \beta x_{i}^{2}}} \\
& +\frac{(b-1)}{2} \sum_{i=1}^{n} \frac{c x_{i}^{2} \mathrm{e}^{-1 / 2 x_{i}\left(\beta x_{i}+2 \alpha\right)} B_{2}}{\left(-1+\mathrm{e}^{-1 / 2 x_{i}\left(\beta x_{i}+2 \alpha\right)}\right)\left(-1+\left(\left(\mathrm{e}^{\alpha x_{i}}-\mathrm{e}^{-1 / 2 \beta x_{i}{ }^{2}}\right) \mathrm{e}^{-\alpha x_{i}}\right)^{\theta c}\right)^{2}}
\end{aligned}
$$

286

Pak.j.stat.oper.res. Vol.X No.3 2014 pp267-288 


$$
\begin{aligned}
& B_{2}=\left(\left(\mathrm{e}^{\alpha x_{i}}-\mathrm{e}^{-1 / 2 \beta x_{i}^{2}}\right) \mathrm{e}^{-\alpha x_{i}}\right)^{\theta c} c \ln \left(1-\mathrm{e}^{-1 / 2 x_{i}\left(\beta x_{i}+2 \alpha\right)}\right) \theta \\
& +\left(\left(\mathrm{e}^{\alpha x_{i}}-\mathrm{e}^{-1 / 2 \beta x_{i}^{2}}\right) \mathrm{e}^{-\alpha x_{i}}\right)^{\theta c}-\left(\left(\mathrm{e}^{\alpha x_{i}}-\mathrm{e}^{-1 / 2 \beta x_{i}^{2}}\right) \mathrm{e}^{-\alpha x_{i}}\right)^{2 \theta c} \\
& V_{\beta a}=\theta c \sum_{i=1}^{n} 1 / 2 \frac{x_{i}^{2} \mathrm{e}^{-\alpha x_{i}-1 / 2 \beta x_{i}^{2}}}{1-\mathrm{e}^{-\alpha x_{i}-1 / 2 \beta x_{i}^{2}}} \\
& V_{\beta b}=\frac{\theta c}{2} \sum_{i=1}^{n} \frac{\left(1-\mathrm{e}^{-1 / 2 x_{i}\left(\beta x_{i}+2 \alpha\right)}\right)^{\theta c-1} x_{i}^{2} \mathrm{e}^{-1 / 2 x_{i}\left(\beta x_{i}+2 \alpha\right)}}{\left.-1+\left(1-\mathrm{e}^{-1 / 2 x_{i}\left(\beta x_{i}+2 \alpha\right)}\right)\right)^{\theta c}} \\
& V_{\beta c}=\theta a \sum_{i=1}^{n} 1 / 2 \frac{x_{i}^{2} \mathrm{e}^{-\alpha x_{i}-1 / 2 \beta x_{i}{ }^{2}}}{1-\mathrm{e}^{-\alpha x_{i}-1 / 2 \beta x_{i}{ }^{2}}}+\frac{b-1}{2} \sum_{i=1}^{n} \frac{\left(1-\mathrm{e}^{-\alpha x_{i}-1 / 2 \beta x_{i}{ }^{2}}\right)^{\theta c} \theta x_{i}{ }^{2} \mathrm{e}^{-\alpha x_{i}-1 / 2 \beta x_{i}{ }^{2}} B_{3}}{\left(-1+\mathrm{e}^{-\alpha x_{i}-1 / 2 \beta x_{i}{ }^{2}}\right)\left(-1+\left(1-\mathrm{e}^{-\alpha x_{i}-1 / 2 \beta x_{i}{ }^{2}}\right)^{\theta c}\right)^{2}} \\
& B_{3}=-c \ln \left(1-\mathrm{e}^{-\alpha x_{i}-1 / 2 \beta x_{i}^{2}}\right) \theta+2\left(1-\mathrm{e}^{-\alpha x_{i}-1 / 2 \beta x_{i}^{2}}\right)^{\theta c} \theta c \ln \left(1-\mathrm{e}^{-\alpha x_{i}-1 / 2 \beta x_{i}^{2}}\right) \\
& +1-\left(1-\mathrm{e}^{-\alpha x_{i}-1 / 2 \beta x_{i}^{2}}\right)^{\theta c} \\
& V_{\theta \theta}=-\frac{n}{\theta^{2}}-(b-1) \sum_{i=1}^{n} \frac{\left.\left(1-\mathrm{e}^{-1 / 2 x_{i}\left(\beta x_{i}+2 \alpha\right)}\right)\right)^{\beta c}\left(\ln \left(1-\mathrm{e}^{-1 / 2 x_{i}\left(\beta x_{i}+2 \alpha\right)}\right)\right)^{2} c^{2}}{\left.\left(-1+\left(1-\mathrm{e}^{-1 / 2 x_{i}\left(\beta x_{i}+2 \alpha\right)}\right)\right)^{\theta c}\right)^{2}} \\
& V_{\theta a}=c \sum_{i=1}^{n} \ln \left(1-\mathrm{e}^{-\alpha x_{i}-1 / 2 \beta x_{i}^{2}}\right) \\
& V_{\theta b}=c \sum_{i=1}^{n} \frac{\left(1-\mathrm{e}^{-1 / 2 x_{i}\left(\beta x_{i}+2 \alpha\right)}\right)^{\beta c} \ln \left(1-\mathrm{e}^{-1 / 2 x_{i}\left(\beta x_{i}+2 \alpha\right)}\right)}{-1+\left(1-\mathrm{e}^{-1 / 2 x_{i}\left(\beta x_{i}+2 \alpha\right)}\right)^{\theta c}} \\
& V_{\theta c}=a \sum_{i=1}^{n} \ln \left(1-\mathrm{e}^{-\alpha x_{i}-1 / 2 \beta x_{i}^{2}}\right) \\
& +(b-1) \sum_{i=1}^{n}-\frac{\ln \left(1-\mathrm{e}^{-1 / 2 x_{i}\left(\beta x_{i}+2 \alpha\right)}\right) C_{1}}{\left(-1+\left(\left(\mathrm{e}^{\alpha x_{i}}-\mathrm{e}^{-1 / 2 \beta x_{i}^{2}}\right) \mathrm{e}^{-\alpha x_{i}}\right)^{\theta c}\right)^{2}}
\end{aligned}
$$


Ibrahim Elbatal, Faton Merovci, W. Marzouk

$$
\begin{aligned}
& C_{1}=\left(\left(\mathrm{e}^{\alpha x_{i}}-\mathrm{e}^{-1 / 2 \beta x_{i}^{2}}\right) \mathrm{e}^{-\alpha x_{i}}\right)^{\theta c} c \ln \left(1-\mathrm{e}^{-1 / 2 x_{i}\left(\beta x_{i}+2 \alpha\right)}\right) \theta \\
& +\left(\left(\mathrm{e}^{\alpha x_{i}}-\mathrm{e}^{-1 / 2 \beta x_{i}^{2}}\right) \mathrm{e}^{-\alpha x_{i}}\right)^{\theta c}-\left(\left(\mathrm{e}^{\alpha x_{i}}-\mathrm{e}^{-1 / 2 \beta x_{i}^{2}}\right) \mathrm{e}^{-\alpha x_{i}}\right)^{2 \theta c} \\
& V_{a a}=-\frac{n}{(a+b)^{2}}-n \varphi^{\prime}(a) \\
& V_{a b}=-\frac{n}{(a+b)^{2}} \\
& V_{a c}=\theta \sum_{i=1}^{n} \ln \left(1-\mathrm{e}^{-\alpha x_{i}-1 / 2 \beta x_{i}^{2}}\right) \\
& V_{b b}=-\frac{n}{(a+b)^{2}}+\frac{n}{b^{2}} \\
& V_{b c}=\theta \sum_{i=1}^{n} \frac{\left(1-\mathrm{e}^{-1 / 2 x_{i}\left(\beta x_{i}+2 \alpha\right)}\right)^{\theta c} \ln \left(1-\mathrm{e}^{-1 / 2 x_{i}\left(\beta x_{i}+2 \alpha\right)}\right)}{\left.-1+\left(1-\mathrm{e}^{-1 / 2 x_{i}\left(\beta x_{i}+2 \alpha\right)}\right)\right)^{\theta c}} \\
& V_{c c}=-\frac{n}{c^{2}}-(b-1) \sum_{i=1}^{n} \frac{\left(1-\mathrm{e}^{-1 / 2 x_{i}\left(\beta x_{i}+2 \alpha\right)}\right)^{\theta c}\left(\ln \left(1-\mathrm{e}^{-1 / 2 x_{i}\left(\beta x_{i}+2 \alpha\right)}\right)\right)^{2} \theta^{2}}{\left.\left(-1+\left(1-\mathrm{e}^{-1 / 2 x_{i}\left(\beta x_{i}+2 \alpha\right)}\right)\right)^{\theta c}\right)^{2}}
\end{aligned}
$$

288

Pak.j.stat.oper.res. Vol.X No.3 2014 pp267-288 